\title{
The Hippo tumor suppressor pathway: a report on 'the second workshop on the Hippo tumor suppressor pathway'
}

\author{
H McNeill ${ }^{*, 1,2}$, M Sudol $^{3}$, G Halder ${ }^{4,5}$, S Strano ${ }^{6}$, G Blandino $^{7}$ and $Y$ Shaul ${ }^{*, 8}$ \\ Cell Death and Differentiation (2011) 18, 1388-1390; doi:10.1038/cdd.2011.61; published online 13 May 2011
}

The Second Hippo Tumor Suppressor Pathway Workshop, Ariccia, Rome, 2-5 November 2010

The Second Workshop on the Hippo (Hpo) pathway was held last November in exquisite Chigi Palace in Ariccia, in the outskirts of Rome. All participants were amazed by the rapid progress in the field (Figure 1). The Hpo kinase cascade, first identified in Drosophila is conserved in mammals, and has pivotal roles in the regulation of cell proliferation and apoptosis. In Drosophila, the Ste20 kinase Hpo with its adapter Salvador (Sav) phosphorylates and activates the Warts kinase (Wts), which with its adapter Mats phosphorylates and inhibits the transcriptional co-activator Yorkie. The FERM domain proteins Expanded (Ex) and Merlin (Mer), along with the cell adhesion molecule Fat regulate pathway activity. An analogous pathway exists in mammals, in which the kinase Mst1/2 phosphorylates and activates Lats1/2, which then phosphorylates and inhibits the transcriptional coactivators YAP and TAZ. The meeting expanded our understanding of how this pathway functions.

Although cell contact has been linked to growth control by the Hpo pathway, it has been poorly understood how cell contact information is communicated to this pathway. Substantial progress in this area has been made in the past year. Duojia Pan (MD, USA) reported that Mer controlled Hpo pathway activity in mammals and demonstrated that loss of Mer led to liver cancer. He discussed Kibra as a tumor suppressor that functions together with Ex and Mer to regulate $\mathrm{Hpo}$ kinase cassette, in part by recruiting the latter to the cell membrane. Pan also discussed Drosophila Crumbs (Crb), an apical-basal polarity determinant, as a cell surface protein that regulates $\mathrm{Hpo}$ signaling via direct interactions with Ex. Jeff Wrana (Toronto, Canada) reported that the cytoplasmic localization of Taz/Yap in high-density cells sequesters
SMAD complexes, thereby suppressing TGF- $\beta$ signaling. Furthermore, biochemical analysis showed that the Crb polarity complex (Amot, PatJ, Pals1, Lin 7c) interacts with TAZ/YAP, relaying cell density information by promoting Taz/ Yap cytoplasmic retention and suppressing TGF- $\beta$ signaling. Lotte Angus (St. Andrews, Scotland) reported that Willin/ FRMD6, the ortholog of Ex, binds Ezrin directly and NF2/Mer indirectly. Surprisingly, Willin cannot replace Ex in Drosophila. Fernardo Carmago (MA, USA) uncovered a link between Yap and the adherens junction protein $\alpha$-catenin. In an $\alpha$-catenin knockout, Yap is localized in the nucleus, whereas overexpression of $\alpha$-catenin recruits Yap to adherens junctions. Expression of Yap can rescue $\alpha$-catenin growth defects. Radhika Jagannathan (MI, USA) reported on negative regulation of the Hpo pathway by the Ajuba LIM proteins (Ajuba, LIMD1, and WTIP). Drosophila djub-deficient tissues phenocopy Yki mutants and Ajuba LIM proteins/dJub interact with both LATS/Wts and WW45/Sav to inhibit phosphorylation of YAP/Yki. Another link between cell contacts and regulation of the Hpo pathway is the junctional protein Aspp2. Xin Lu (Oxford, UK) discussed the interaction between Aspp2 and Par3 in the regulation of cell polarity and cell proliferation.

Another emerging link between the cell surface and Hpo pathway activity is the Angiomotin (Amot) family. Angiomotins are angiostatin-binding proteins promoting endothelial cell migration and angiogenesis. Kun-Liang Guan (San Diego, CA, USA) reported that YAP/TAZ interact with Amot. Binding to Amot family proteins is crucial to the tight junction localization of YAP/TAZ, and is also important for their phosphorylation. AmotL2 knockdown results in loss of cell contact inhibition in a manner dependent on the functions of

\footnotetext{
${ }^{1}$ Samuel Lunenfeld Research Institute, Mount Sinai Hospital, Toronto, Ontario, Canada; ${ }^{2}$ Department of Molecular Genetics, University of Toronto, Toronto, Ontario, Canada; ${ }^{3}$ Laboratory of Signal Transduction and Proteomic Profiling Weis, Center for Research, Geisinger Clinic, 100 North Academy Avenue, Danville, PA, USA; ${ }^{4}$ Department of Biochemistry and Molecular Biology, University of Texas MD Anderson Cancer Center, Houston, TX, USA; ${ }^{5}$ Program in Developmental Biology, Baylor College of Medicine, Houston, TX, USA; ${ }^{6}$ Molecular Chemioprevention Unit, Scientific Director Department, Istituto Nazionale Tumori Regina Elena IRCCS, Rome, Italy; ${ }^{7}$ Translational Oncogenomic Unit, Regina Elena Cancer Institute, Rome, Italy and ${ }^{8}$ Department of Molecular Genetics, Weizmann Institute of Science, Rehovot, Israel ${ }^{*}$ Corresponding authors: H McNeill, Samuel Lunenfeld Research Institute, Mount Sinai Hospital, 600 University Avenue, Room 884, Toronto, Ontario M5G 1X5, Canada. Tel: + 1416586 8267; Fax: + 1416586 8588; E-mail: mcneill@lunenfeld.ca or Y Shaul, Department of Molecular Genetics, Weizmann Institute of Science, Rehovot 76100, Israel. Tel: 9728934 2320; Fax: 97289344108 ; E-mail: yosef.shaul@weizmann.ac.il
} 
a

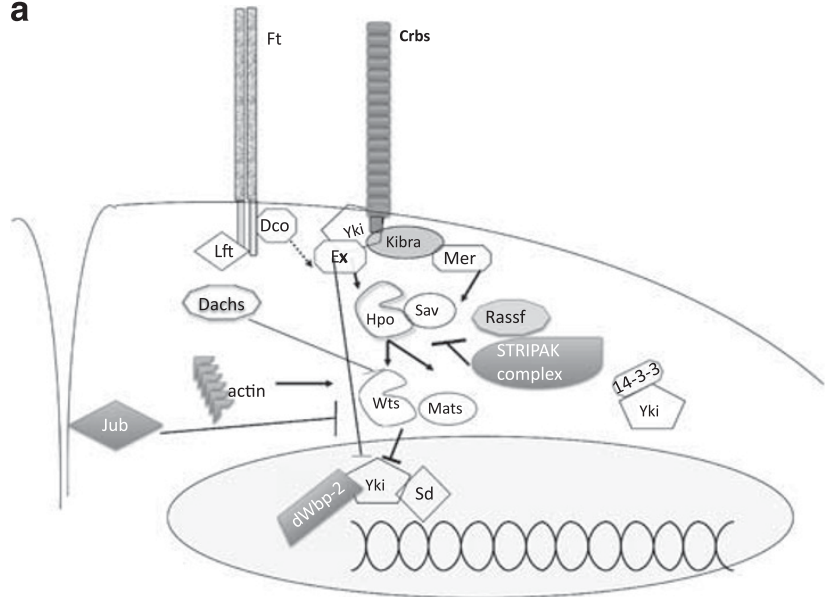

b

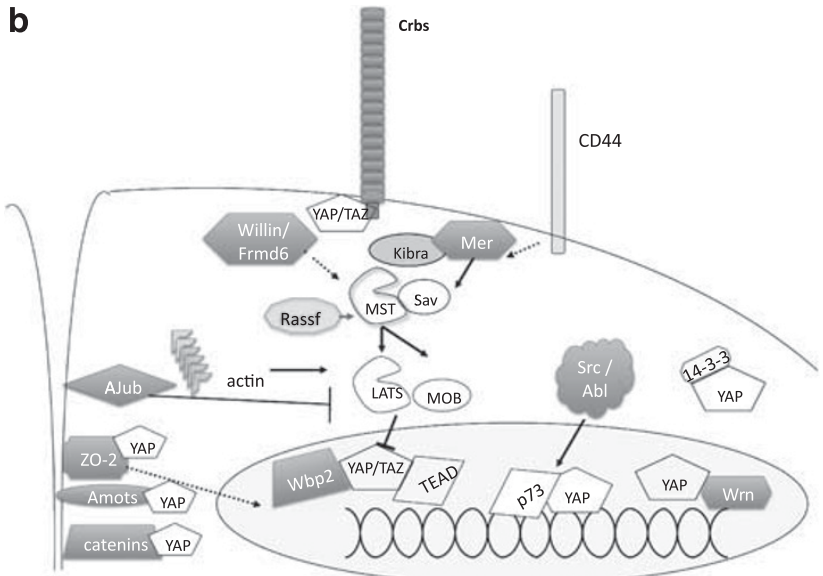

Figure 1 New additions to the Hippo pathway in flies (a) and man (b). In white are components of the Hippo pathway familiar to attendees of the first Hippo workshop in 2009. In blue are new components reported at the second Hippo workshop in 2010, discussed in this report. Notable are the large number of components that function upstream of the core kinase cassette, including a number of junction-associated proteins (Amot, Aspp, Ajuba/Jub, ZO-2, Willin/ FRMD6, $\alpha$-catenin, Crumbs). Also notable are new kinases (Src, Abl, JNK) that modulate YAP/Yki signaling, as well as new transcriptional regulators (Wbp2, Wrn). In light blue are components introduced at the last meeting, but whose functions were further elucidated at the 2010 meeting (Kibra, RASSF). The diagrams are not complete, but highlight the new information shared at the latest meeting

YAP/TAZ. SW Chan (Singapore) showed that Wbp2, Amot, and AmotL1 bind TAZ/YAP. Joe Kissel (PA, USA) reported that Amot acts downstream of Merlin/NF2 by direct interaction to support tumor suppression function, suggesting Amot may have different functions in different cell types. A role for Amot was also suggested in mouse development. During preimplantation, embryos establish two distinct cell lineages the trophectoderm (TE) and inner cell mass (ICM). Hiroshi Sasaki (Kumamoto, Japan) reported that in TE, Amot is nuclear and in ICM perinuclear. He suggested that cell polarity regulated by Par6b and PKC, dictates Amot redistribution.

One of the two main kinases of the pathway Mst1/2 (Hpo) was reported to have a role in apoptosis with distinct upstream inputs involving Raf, Ras, PP2A phosphatase, and RASSF1A. Gerald Pfeifer (CA, USA) investigated the mechanism of regulation of MST1/2 by the RASSF1A tumor suppressor.
RASSF1A interacts with MST1/2, inducing autophosphorylation and activation and stabilizes MST2. They found that dephosphorylation of MST1/2 by PP2A phosphatase is prevented by RASSF1A, shifting the balance of MST1/2 to the activated form and resulting in apoptosis. Nick Tapon (London, UK) identified the STRIPAK complex composed of PP2A subunits as a major negative regulator of the Drosophila Hpo pathway. Key components of the STRIPAK complex also include $\mathrm{CCM} 3$ protein and GCKIII kinases, providing new links between the Hpo pathway and other pathways. David Matallanas (Dublin, Ireland) addressed the role of MST2 and activated K-Ras in apoptosis. He suggests that EGF-KRas induces transient activation of Rassf1A-MST2, whereas activated K-Ras V12 induces sustained MST2 activation, which in turn via Lats2 inhibits Mdm2 and activates p53 leading to apoptosis. In accord with this model, colorectal cancer MST2 expression is lost in K-Ras-mutant-positive cases. Eric O'Neill (Oxford, UK) explored the role of MST in DNA damage induced apoptosis. In response to DNA damage, the Brca2 protein binds to Brca1, which regulates Rad51 to fix breaks in DNA. Howard Donninger (KY, USA) reported that Sav may function independently of MST coupling to LATS. Sav is a mediator of RASSF1A induced apoptosis via p73 targets. Donninger identified an RASSF1A point mutant in the SARAH domain that binds to Sav but not to Mst $1 / 2$.

New findings on the regulation of Lats and YAP were also revealed. Yael Aylon (Rehovot, Isreal) reported that Lats2 phosphorylates ASPP1 (apoptosis-stimulating protein of p53-1) and drives its translocation into the nucleus in response to oncogenic stress. Lats2 and ASPP1 shunt p53 to proapoptotic genes and promote death. YAP disrupts Lats2-ASPP1 binding to inhibit apoptosis and to promote the formation of multinucleated cells. Rami Aqelian (Jerusalem, Isreal) revealed a functional link between the E3 ubiquitin ligase Itch and the Hpo pathway. Kenneth Irvine (NJ, USA) discussed the regulation and role of the Drosophila Hpo pathway during regeneration, showing that $\mathrm{Yki}$ is activated in response to tissue damage through the JNK pathway. Subham Basu (London, UK) reported on the JNK-mediated phosphorylation of YAP and its role under UV radiation.

Several links were revealed between the Hpo pathway and mitosis. Amy Bui (MA, USA) reported that Yap and Lats are localized to the mitotic machinery, including the spindle and midbody ring. Loss of Yap affects Rho activity and localization of the central spindle proteins during cytokenesis, giving rise to aneuploidy. Norikazu Yabuta (Osaka, Japan) reported on the axis Aurora-A-Lats2-Aurora-B regulating chromosome segregation. Reto Kohler (Basel, Switzerland) reported that hMOB2 and MST3 inhibit and activate NDR1/2 kinases, respectively, in the G1 phase. MST3 and NDR stabilize p21 by phosphorylation, preventing ubiquitination. Hiroshi Nojima (Osaka, Japan) generated mice lacking the Lats $1 \mathrm{~N}$-terminus, and found that unlike the Last2, Lats1-knockout mice are not embryonic lethal. However, derived MEFs have nuclear YAP, a wide variety of mitotic defects and an oncogenic phenotype. Thus, the $\mathrm{N}$-terminus of Lats 1 is required for chromosomal stability and tumor suppression.

Mhairi Towler (Aberdeen, UK) reported that YAP is involved in myotube formation. In $\mathrm{C} 2 \mathrm{C} 12$ myoblasts, $\mathrm{S} 127$ phosphorylation 
is low and Yap is nuclear in the nucleoli, whereas in differentiated cells S127 phosphorylated Yap accumulates to high level in the cytosol. Interestingly, in mouse satellite cells, Yap levels are low but become upregulated in the MyoD positive cells. Junichi Sadoshima (NJ, USA) reported that Yap stimulates hypertrophy of cardiac myocytes but inhibits apoptosis. Under this condition Yap, via an E-box induces expression of the muscle specific mir-206, which in turn mediates hypertrophy and survival of cardiac myocytes.

Kieran Harvey (Melbourne, Australia) used Drosophila imaginal disks as a model to demonstrate that Hpo pathway activity is repressed during tissue regeneration. He found that in regenerating tissues Yki levels increase, and that dachs is needed for regeneration, thus implicating the Fat branch of the Hpo pathway as a sensor of damaged tissues. Phillip Karpowicz (MA, USA) presented evidence linking Fat and Dachsous to $\mathrm{Yki}$ in the process of acute regeneration of intestinal stem cells. Yutaka Hata (Tokyo, Japan) studied the possible role of Yap and TAZ in establishment of cancer stem cells using Taz S89A and Yap S127A in combination with active Ras.

New links were revealed between the Hpo pathway and ovarian cancer and liver cancer. Kieran Harvey showed that an increased YAP activity correlated with reduced survival in patients suffering ovarian clear cell cancer, independent of grade or stage. Chad Hall (Houston, TX, USA) quantified the ratio between nuclear and cytoplasmic Yap in ovarian cancer and revealed that this ratio correlates with poor survival. Dae-Sik Lim (Daejeon, Korea) generated mice with liver specific ablation of WW45. These mice show an enlarged liver with exclusive expansion of the oval cells that develop hepatoma. David Moore (Houston, TX, USA) demonstrated a link between the nuclear hormone receptors: farnesoid $X$ (FXR) and small heterodimer partner (SHP) and Hpo signaling. FXR and SHP double knockouts display liver defects that are similar to Mst1/2 mutants, with overproliferation and activated YAP. Jim Martin (TX, USA) presented data indicating that $\mathrm{Hpo}$ is important in limiting cardiac muscle proliferative potential.

Uri Gat (Jerusalem, Isreal) provided an evolutionary view of Hpo signaling. Moshe Oren (Isreal), our keynote lecturer, discussed how insights into p53, as a well-understood tumor suppressor, might guide work on the Hpo tumor suppressor pathway. In a closing summary, K-L Guan highlighted the progress made in the Hpo pathway and also raised several important questions, including that of the dual function of YAP as oncogene or pro-apototic protein.

\section{Conflict of Interest}

The authors declare no conflict of interest. 